\title{
OCORRÊNCIA DE Cryptosporidium spp. EM BEZERROS MANTIDOS SOB DOIS DIFERENTES SISTEMAS DE MANEJO
}

\author{
Willian Marinho Dourado Coelho ${ }^{1}$, Vamilton Alvares Santarém ${ }^{2}$, Wilma Aparecida Starke Buzetti ${ }^{1}$, \\ Daniel Andrade Da Silva ${ }^{3}$
}

${ }^{1}$ Universidade Estadual Paulista - UNESP, Ilha Solteira, SP. ${ }^{2}$ Universidade do Oeste Paulista - UNOESTE, Medicina Veterinária, Presidente Prudente, SP. Faculdade de Ciências Agrárias de Andradina - FCAA, Andradina, SP. E-mail: willianmarinho@hotmail.com

\section{RESUMO}

O objetivo deste estudo foi determinar a prevalência de Cryptosporidium spp. em bezerros bovinos mantidos sob sistemas de manejo intensivo, extensivo e correlacionar a presença deste protozoário com a idade e consistência das fezes destes animais. Amostras fecais de 356 bezerros com até um ano de idade, machos e fêmeas, de diferentes raças, foram analisadas por meio das técnicas de Kinyoun modificado e de Sheather. Dos animais avaliados, 54,21\% (193/356) foram destinados à bovinocultura de corte (sistema extensivo) e 45,78\% (163/356) para a extração do leite (sistema intensivo). Presença de Cryptosporidium spp. foi observada em 7,58\% (27/356) dos animais, sendo $2,07 \%(4 / 193)$ e $14,11 \%$ (23/163) em bezerros de corte e leite, respectivamente. Prevalência mais alta de oocistos foi observada em animais com idade inferior a 15 dias, com a presença de diarréia, considerada estatisticamente significativa. Os resultados permitem inferir que o manejo do gado foi fator importante para a ocorrência deste parasito, favorecida pelo sistema de criação intensivo adotado na produção de leite predominantemente em animais com idade inferior a 15 dias, com manifestação de sinais clínicos de diarréia.

Palavras-chave: bovinos, criptosporidiose, diarréia, manejo, fatores de risco

\section{OCCURRENCE OF Cryptosporidium spp. IN CALVES KEPT UNDER TWO DIFFERENT MANAGEMENT SYSTEMS}

\begin{abstract}
The aim of this study was to determine the prevalence of Cryptosporidium spp. in cattle calves kept under intensive and extensive management systems, and correlate the presence of this protozoan with age and fecal consistency of these animals. Stool samples from 356 calves up to one year of age, males and females, different breeds, were analyzed by techniques of modified Kinyoun and Sheather. Of all animals, 54.21\% (193/356) were intended for beef cattle (extensive system) and $45.78 \%$ (163/356) for milk extraction (intensive system). Presence of Cryptosporidium spp. were observed in $7.58 \%(27 / 356)$ of animals, being $2.07 \%(4 / 193)$ and $14.11 \%(23 / 163)$ in beef calves and milk, respectively. Higher prevalence of oocysts was observed in animals aged less than 15 days, with the presence of diarrhea, which highlighted a statistically significant difference. Through these results we can infer that the management of livestock was an important factor for the occurrence of this parasite, with this type of infection favored by intensive breeding system adopted in dairy farming, occurring predominantly in animals aged less than 15 days and clinical signs of diarrhea.
\end{abstract}

Keywords: cattle, cryptosporidiosis, diarrhea, management, risk factors 


\section{INTRODUÇÃO}

Diarréia em bezerros é uma doença multifatorial, resultante da interação entre os agentes etiológicos (Langoni et al., 2004), estado imunológico e manejo do rebanho (Benesi, 1999), especialmente em ambientes com superlotação de animais (Carneiro et al., 1988). Entre estes agentes, o gênero Cryptosporidium se destaca nas infecções gastrointestinais, sendo referido como uma das principais causas de distúrbios gastrointestinais em bovinos jovens (Oliveira Filho et al., 2007).

\section{OBJETIVO}

O objetivo deste estudo foi determinar a prevalência de Cryptosporidium spp. em bezerros mantidos em sistemas de manejo intensivo e extensivo, e correlacionar a presença do protozoário com a idade e consistência fecal destes animais.

\section{METODOLOGIA}

As fezes foram coletadas diretamente do reto de 356 bezerros machos e fêmeas de diferentes raças, com até um ano de idade. Estes animais foram provenientes de diferentes fazendas localizadas nos estados de Goiás, Minas Gerais, Mato Grosso do Sul e São Paulo, Brasil. Foi utilizada a flotação em solução de sacarose (gravidade específica de 1,20 g / mL) (Pratt, 1997) e de Kinyoun modificado (MacPherson \& McQueen, 1993), para concentração e coloração de oocistos, respectivamente. As amostras de fezes foram classificadas de acordo com a consistência. A frequência segundo aptidão zootécnica foi analisada usando teste qui-quadrado, adotando nível de significância de 5\%. Este estudo foi aprovado pelo Comitê de Ética em Experimentação Animal da Faculdade de Ciências Agrárias e Veterinárias da UNESP- Jaboticabal, São Paulo, Brasil, protocolo no. 005589-09

\section{RESULTADOS}

Oocistos de Cryptosporidium spp. foram observados em 7,58\% (27/356) dos animais sendo, $2,07 \%$ (4/193) em bezerros criados extensivamente, destinados ao produção de carne e 14,11\% (23/163) em bezerros criados intensivamente destinados a exploração leiteira. Prevalência mais alta de oocistos foi observada em animais com idade inferior a 15 dias e com manifestação diarreia, com diferenças estatisticamente significativas (Fig. 1). Não foi observada diferença estatistica significativa $p \geq 0,05$ entre sexo e padrões raciais na prevalência de Cryptosporidium spp. A maior porcentagem de animais positivos para este parasito foi observada em gado Holandês (Tab.1). 


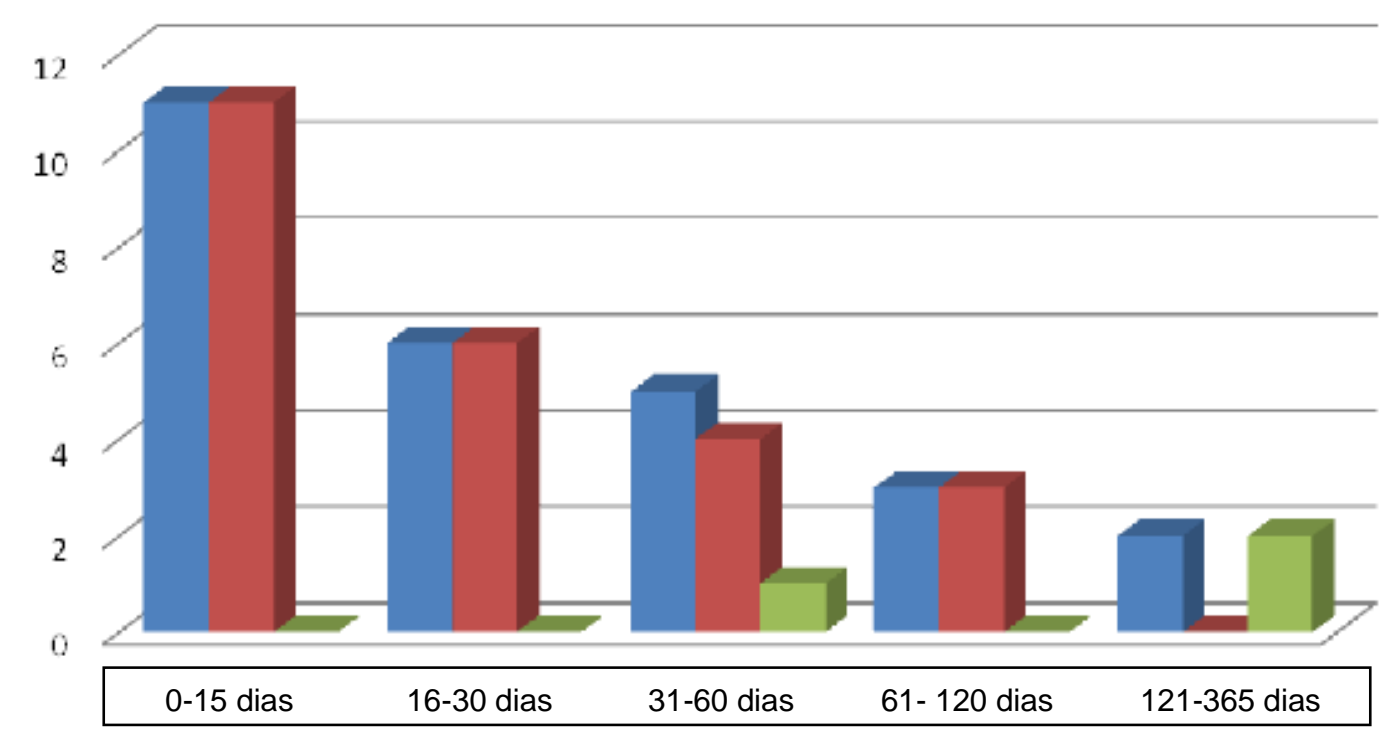

Animais positivos Animais com diarréia Animais sem Diarréia

Figura 01. Distribuição dos 27 bezerros positivos para o Cryptosporidium spp. de acordo com a idade e consistência das amostras de fezes.

Tabela 01. Prevalência de Cryptosporidium spp. em bezerros de corte e de leite, de acordo com o tipo de criação e padrões raciais.

\begin{tabular}{ccccc}
\hline Tipo de criação & Raça & Negativo & Positivo & Total \\
\hline & Gir leiteiro & $4(80 \%)$ & $1(20 \%)$ & 5 \\
& Holandês & $60(78,95 \%)$ & $16(21,05 \%)$ & 76 \\
Bezerros de corte & Jersey & $2(100 \%)$ & $0(0,00 \%)$ & 2 \\
(Sistema extensivo) & Mestiço & $69(93,24 \%)$ & $5(6,76 \%)$ & 74 \\
& Pardo suíço & $5(83,33 \%)$ & $1(16,67 \%)$ & 6 \\
\hline Bezerros de leite & Mestiço & $91(97,85 \%)$ & $2(2,15 \%)$ & 93 \\
(Sistema intensivo) & Nelore & $98(98 \%)$ & $2(2 \%)$ & 100 \\
\hline
\end{tabular}

\section{DISCUSSÃO}

Valores de prevalência mais altos (Almeida et al., 2008) e mais baixos (Cardoso et al., 2008) aos observados neste estudo foram relatados em bezerros bovinos provenientes dos estados do Rio de Janeiro e São Paulo, Brasil, com 61\% (61 / 100) e 0,6\% (5/849) das amostras positivas para Cryptosporidium spp. No estudo de Almeida et al. (2008), não houve diferenças significativas entre os sistemas de criação extensivos e semi-intensivos. Este mesmo evento foi relatado por Garcia;Lima, (1994) no rebanho bovino leiteiro no estado de Minas Gerais, Brasil. Em contraste, os resultados observados em nosso estudo sugerem prevalência mais alta em sistemas intensivos de criação de gado de exploração leiteira, nos quais é constante a superlotação de pastagens, em comparação com o gado criado extensivamente, que apenas é recolhido a pontos de aglomeração em determinados momentos do manejo da cultura nas propriedades.

Em nosso estudo, a maioria dos animais infectados com Cryptosporidium apresentou sinais clínicos de enterite. Da mesma forma, Oliveira Filho et al. (2007) observaram que, o maior número de casos de diarréia em bezerros da raça Nelore, criados extensivamente ocorreu principalmente devido à infecção Cryptosporidium. Em desacordo com os nossos resultados, na obra de Cardoso et al. (2008) não foi observado a manifestação clínica da diarreia em bezerros, embora tenham sido positivos para Cryptosporidium spp. Foi expedido em nosso estudo que o pico da infecção por Cryptosporidium ocorreram em animais com até 15 dias de vida, diminuindo gradualmente com o 
avançar da idade. Corroborando nossos achados, Langoni et al. (2004) observaram incidência mais alta de Cryptosporidium em bezerros com idade entre oito e 14 dias. Desta forma, animais com idade inferior a dois meses de idade foram mais susceptíveis à infecção por Cryptosporidium (Maldonado-Camargo et al., 1998;. Castro-Hermida et al., 2002).

\section{CONCLUSÃO}

Os resultadossugeferem que o sistema de criação dos animais é um fator importante para a ocorrência de Cryptosporidium spp., sendo a infecção favorecida pelo sistema de criação intensiva adotada na pecuária leiteira, ocorrendo predominantemente em animais com idade inferior a 15 dias e com manifestações clínicas de diarreia.

\section{REFERÊNCIAS BIBLIOGRÁFICAS}

ALMEIDA, A.J.; OLIVEIRA, F.C.R.; TEIXEIRA, C.S. Risco relativo da infecção por parasitos do gênero Cryptosporidium em bezerros bovinos no Norte do Estado do Rio de Janeiro, Brasil. Revista Brasileira de Parasitologia Veterinária, v.17, n.1, p.243-248, 2008.

BENESI, F.J. Síndrome diarréia dos bezerros. Revista do CRMV-ES, v. 2, p. 10-13, 1999.

CARDOSO, J.M.S.; SILVEIRA, F.L.; ARAÚJO, A.J.U.S.; DE CARVALHO, J.C.C.; KANAMURA, H.Y. Ocorrência de Cryptosporidium spp. em um rebanho bovino leiteiro no Município de Caçapava, Estado de São Paulo, Brasil. Revista Brasileira de Parasitologia Veterinária, v.17, n.1, p.239-242, 2008.

CARNEIRO, J.R.; CAMPOS, D.B.; LINHARES, G.C.; RODRIGUES, N. Eimeria em bovinos mestiços Zebu-Holandês procedentes da bacia leiteira de Goiânia. Arquivo Brasileiro de Medicina Veterinária e Zootecnia, v.40, n.6, p.355-360, 1988.

CASTRO-HERMIDA, J.A.; GONZÁLES-LOSADA, Y.A.; ARES-MAZÁS, E. Prevalence of and risk factors involved in the spread of neonatal bovine cryptosporidiosis in Galicia (NW Spain). Veterinary Parasitology, v.106, n1, p.1-10, 2002. https://doi.org/10.1016/S0304-4017(02)00036-5

GARCIA, A.M. LIMA, J.D. Prevalência de Cryptosporidium spp. em Rebanhos leiteiros de Pará de Minas (MG) e sua relação com práticas de manejo. Revista Brasileira de Parasitologia Veterinária, v.3, n.1, p.23-28, 1994.

LANGONI, H.; LINHARES, A.C.; ÁVILA, F.A.; DA SILVA, A.V.; ELIAS, A.O. Contribution to the study of diarrhea etiology in neonate dairy calves in São Paulo state, Brazil. Brazilian Journal of Veterinary Research and Animal Science, v.41, n.5, p.313-319, 2004. https://doi.org/10.1590/S1413$\underline{95962004000500004}$

MAC-PHERSON, D.W.; MCQUEEN, R. Cryptosporidiosis: Multiattribute evaluation of six diagnostic methods. Journal of Clinical Microbiology, v.31, p.198-202, 1993.

MALDONADO-CAMARGO, S.; ATWILL, E.R.; SALTIJERAL-OAXACA, J.A.; HERRERA-ALONSO, L.C. Prevalence of and rik factors for shedding of Cryptosporidium parvum in Holstein Freisian dairy calves in central Mexico. Preventive Veterinary Medicine, v.36, n.2, p.95-107, 1998. https://doi.org/10.1016/S0167-5877(98)00084-1 
OLIVEIRA FILHO, J.P.; SILVA, D.P.G.; PACHECO, M.D.; MASCARINI, L.M.; RIBEIRO, M.G.; ALFIERI, A.A.; ALFIERI, A.F.; STIPP, D.T.; BARROS, B.J.P.; BORGES, A.S. Diarréia em bezerros da raça Nelore criados extensivamente: estudo clínico e etiológico. Pesquisa Veterinária Brasileira, v.27,n.10, p.419-424, 2007. https://doi.org/10.1590/S0100-736X2007001000006

PRATT, P.W. Laboratory procedures for veterinary technicians. 3ed. Saint Louis: Mosby,1997. 